\title{
Long-term survival after concurrent chemoradi- ation therapy for esophageal cancer with tracheal invasion
}

\author{
Joon Hyun Cho and Tae Nyeun Kim
}

Division of Gastroenterology and Hepatology, Department of Internal Medicine, Yeungnam University College of Medicine, Daegu, Korea

Received: January 20, 2015

Revised : May 28, 2015

Accepted: June 15, 2015

\section{Correspondence to}

Tae Nyeun Kim, M.D.

Division of Gastroenterology and Hepatology, Department of Internal Medicine, Yeungnam University College of Medicine, 170 Hyeonchung-ro, Nam-gu, Daegu 42415 , Korea

Tel: $+82-53-620-3842$

Fax: +82-53-654-8386

E-mail: tnkim@yu.ac.kr
To the Editor,

Esophageal cancer is a highly lethal malignancy with poor survival outcome, and usually presents in advanced stage due to its anatomical characteristics. Treatment of esophageal cancer with airwayinvasion continues to present difficult management decisions. In these patients, surgery is not considered for the treatment modality because operative complications are considerable and the prognosis is poor, even if radical esophageal dissection may be achieved by combined excision of the trachea. Although long-term survival has been reported in a minority of patients with $\mathrm{T}_{4}$ esophageal cancer after combined chemoradiation therapy, such cases have also been associated with worsening or development of new fistulas as well as treatment-related deaths in a significant proportion of patients [1]. Here we report on a case of a patient with advanced esophageal squamous cell carcinoma with tracheal invasion treated by concurrent chemoradiotherapy, resulting in sustained complete regression.

A 67-year-old man complained of dysphagia for over 2 months. Upper gastrointestinal endoscopic examination performed in a local hospital showed an ulcerofungating mass in the mid esophagus. He was referred to our hos- pital for further examination and treatment, and admitted in December, 2006. He was a heavy drinker (more than 30 years) and a heavy smoker (40 cigarettes/ day for approximately 40 years). He had no notable medical history. Laboratory data including tumor markers were all within normal limits. Endoscopy showed a circumferential luminal protruding ulcerative mass located between 23 and $30 \mathrm{~cm}$ distal from the incisors (Fig. 1A). Histology of a biopsy specimen indicated that it was a well differentiated squamous cell carcinoma. A position emission tomography/computed axial tomography (PET/CT) scan demonstrated marked fludeoxyglucose (FDG) uptake in the mid esophageal mass (maximal standard uptake value [SUVmax] 32.0) with tracheal invasion and regional metastatic lymphadenopathy (SUVmax 12.7) without evidence of distant metastasis (Fig. 2A). A CT scan of the chest showed no finding indicating a tracheoesophageal fistula (Fig. 3). Based on these findings, we made a diagnosis of esophageal cancer with invasion of trachea and regional lymph node metastasis, classified as esophageal cancer, cStage IIIC: T4bNiMo, according to the tumor, node, metastasis (TNM) staging system of the American Joint Committee on Cancer (AJCC) and the International Union Against Cancer 

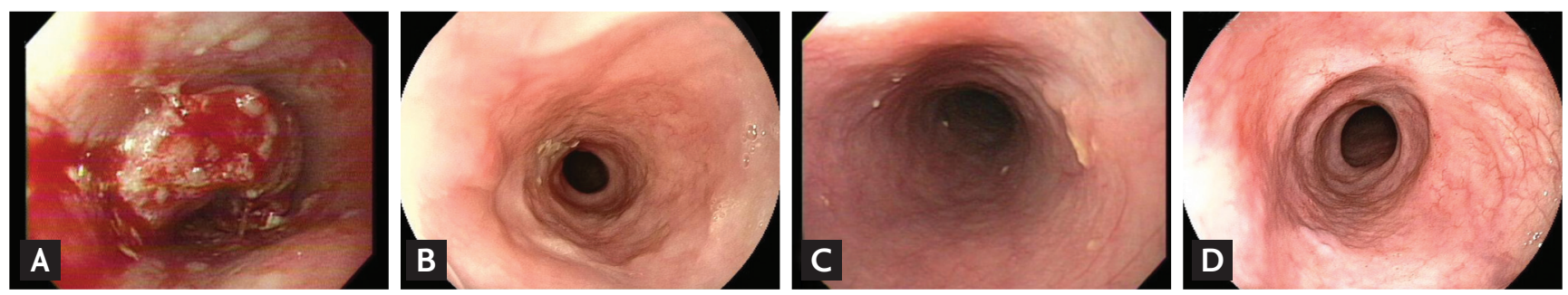

Figure 1. Upper gastrointestinal endoscopic images: (A) baseline, (B) after three cycles of chemoradiotherapy, (C) after completion of chemoradiotherapy, and (D) 6 years after completion of chemoradiotherapy.
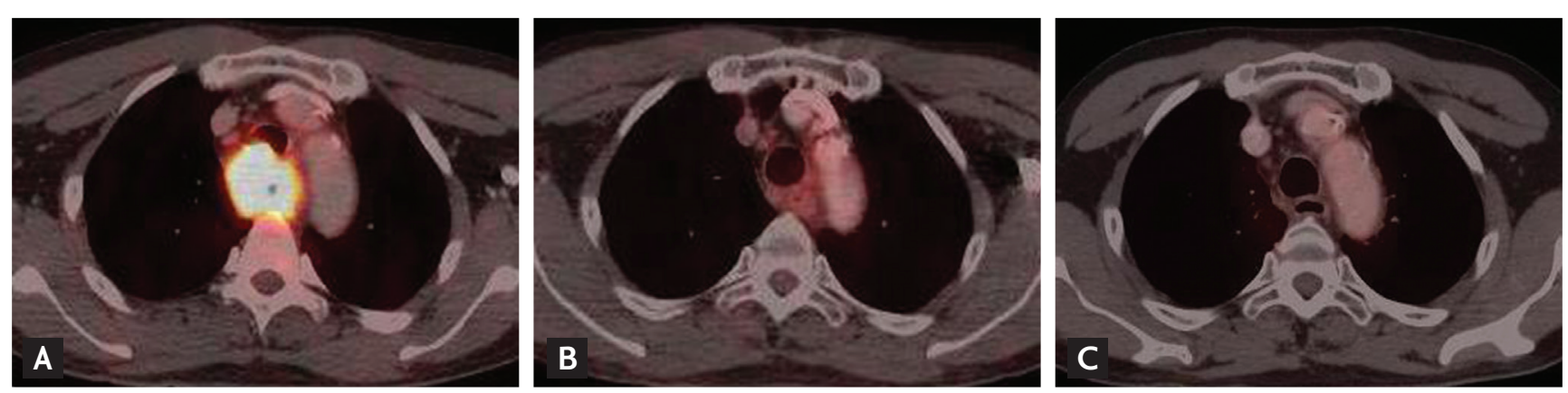

Figure 2. Position emission tomography/computed axial tomography images: (A) baseline, (B) after three cycles of chemoradiotherapy, and $(\mathrm{C}) 3$ years after completion of chemoradiotherapy.

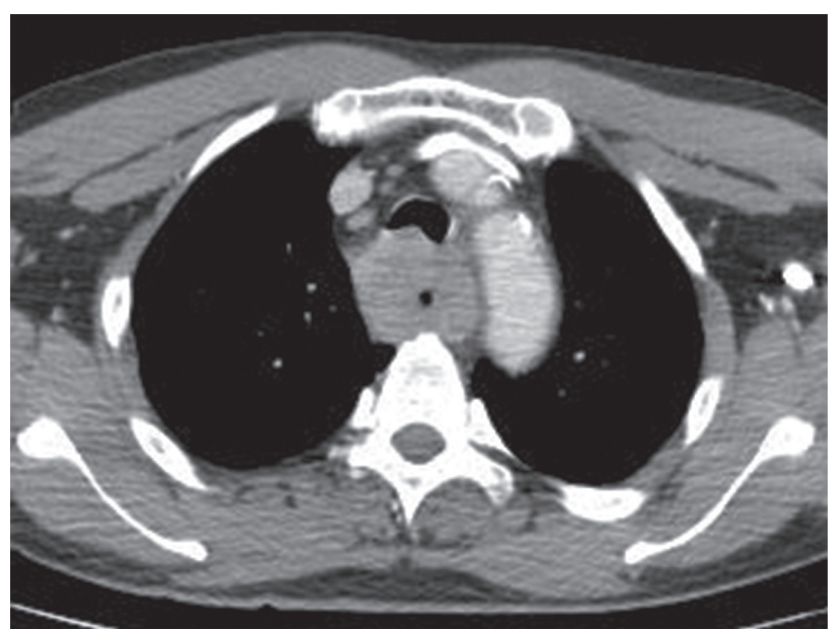

Figure 3. Computed tomography image at baseline.

(UICC) [2], and decided to perform concurrent chemoradiation therapy with his informed consent.

The regimen of chemotherapy consisted of 5-fluorouracil (5-FU) and cisplatin. Continuous infusion of 5-FU was administered at a daily dose of $750 \mathrm{mg} / \mathrm{m}^{2}$ on days 1 to 4 every 4 weeks, and infusion of cisplatin was administered over a 2-hour-period at a daily dose of $60 \mathrm{mg} / \mathrm{m}^{2}$ on day 1 every 4 weeks; this regimen was repeated over six cycles for about 6 months with concurrent radiotherapy at a daily fractional dose of 2 Gy five times per week over 5 weeks (total dose $50 \mathrm{~Gy}$ ). After three cycles of concurrent chemoradiation therapy, the patient showed complete resolution of dysphagia. A repeat PET/CT scan showed no visible hypermetabolic mass on mid esophagus and mild FDG uptake on locoregional lymph node (SUVmax 3.7) (Fig. 2B). Endoscopy also showed no stenotic mass lesion except an ulcer scar on the previous lesion site (Fig. 1B). Throughout the course of chemoradiation therapy, there was no development of significant toxicity except mild radiation pneumonitis.

After completion of therapy, follow-up endoscopy showed no tumor at the previous site of the tumor lesion (Fig. 1C). Thereafter, he underwent periodic clinical and instrumental checks, which were always negative. It is now about 8 years since his first treatment, and the patient is still alive and disease-free. The most recent images of endoscopy and PET/CT scan are shown in Figs. 1D and $2 \mathrm{C}$, respectively.

In our case report, the patient was diagnosed with tracheal invasion ( $\mathrm{cT}_{4} \mathrm{~b}$ ) based on the finding of PET/CT scan, although pathologic examination by bronchoscopy was not performed. In the latest revision of the combined AJCC/UICC TNM staging criteria, unresectable $\mathrm{T}_{4}\left(\mathrm{~T}_{4} \mathrm{~b}\right)$ primary disease includes invasion of the aorta, 
trachea, heart, great vessels, or the presence of a tracheoesophageal fistula. Invasion of the pleura, pericardium, or diaphragm is classified as T4a disease and considered potentially resectable disease.

For patients with locally advanced esophageal cancer, a significant improvement in local control and overall survival was achieved with concurrent chemoradiotherapy compared with radiotherapy alone, and concurrent chemoradiotherapy consisting of 5 -FU and cisplatin is now considered the standard therapy for patients with esophageal cancer [3]. Several studies reported that concurrent chemoradiotherapy was also effective for patients with unresectable $\mathrm{T}_{4}$ esophageal cancer [1]; however, there has been substantial controversy regarding its feasibility and effectiveness for $\mathrm{T}_{4}$ tumors invading adjacent structures. In addition, esophageal cancer with airway invasion with or without fistula presents a challenging therapeutic dilemma with no standard therapy, and patients who undergo radiotherapy are at risk for worsening or development of new esophagorespiratory fistulas and esophageal perforation. In our case report, there was no development of significant toxicity except mild radiation pneumonitis throughout the course of chemoradiotherapy, although we were highly concerned about the formation of a tracheoesophageal fistula due to concurrent radiotherapy.

The benefit of surgery after concurrent chemoradiotherapy is controversial, particularly for $\mathrm{T}_{4}$ esophageal cancer. A recent randomized trial (which included patients with $\mathrm{T}_{4}$ tumors) directly comparing chemoradiotherapy alone to chemoradiotherapy followed by surgery failed to demonstrate better survival benefit, although better locoregional control and a lesser need for palliative procedures was observed for both when surgery was a component of multimodality treatment [4]. The patient populations in both were either exclusively or predominantly squamous cell carcinoma. These results suggest that in patients with locally advanced esophageal cancer, particularly squamous cell carcinoma, surgery may be omitted in those who responded well to chemoradiation.

Only a few cases of long-term survival after chemoradiotherapy in patients with advanced esophageal cancer with airway invasion have been reported [5]. To the best of our knowledge, this case showed the longest survival.
Our experience suggests that although the prognosis of advanced esophageal cancer with invasion of other organs is usually poor, concurrent chemoradiotherapy can sometimes have a dramatic effect in some patients.

Keywords: Esophageal neoplasms; Chemoradiotherapy; Long-term survival

\section{Conflict of interest}

No potential conflict of interest relevant to this article was reported.

\section{REFERENCES}

1. Nishimura Y, Suzuki M, Nakamatsu K, Kanamori S, Yagyu Y, Shigeoka H. Prospective trial of concurrent chemoradiotherapy with protracted infusion of 5-fluorouracil and cisplatin for $\mathrm{T}_{4}$ esophageal cancer with or without fistula. Int J Radiat Oncol Biol Phys 2002;53:134139.

2. Rice TW, Rusch VW, Ishwaran H, Blackstone EH; Worldwide Esophageal Cancer Collaboration. Cancer of the esophagus and esophagogastric junction: data-driven staging for the seventh edition of the American Joint Committee on Cancer/International Union Against Cancer Cancer Staging Manuals. Cancer 2010;116:37633773 .

3. Minsky BD, Neuberg D, Kelsen DP, et al. Final report of Intergroup Trial o122 (ECOG PE-289, RTOG 90-12): phase II trial of neoadjuvant chemotherapy plus concurrent chemotherapy and high-dose radiation for squamous cell carcinoma of the esophagus. Int J Radiat Oncol Biol Phys 1999;43:517-523.

4. Stahl M, Stuschke M, Lehmann N, et al. Chemoradiation with and without surgery in patients with locally advanced squamous cell carcinoma of the esophagus. J Clin Oncol 2005;23:2310-2317.

5. Kinoshita Y, Udagawa H, Tsutsumi K, et al. Esophageal carcinoma with tracheal stenosis due to tumor invasion and long survival after chemoradiotherapy. Dis Esophagus 2003;16:261-264. 